intraocular acrylic lenses increase the death rate of endothelial cells (as does any intraocular procedure) to an extent which may irreversibly cloud the cornea. Cystoid oedema of the macula is commoner when such lenses are used than in the simple cataract extraction. Many operative and postoperative mishaps seem to be associated with the use of intraocular lenses, and the number of modified varieties testifies that something is still amiss with the whole principle.

Extended-wear soft contact lenses, on the other hand, are probably much less prone to give rise to disastrous complications, provided they are well-fitted and are of the high water content type. Even so, a patient with soft lenses should have frequent and prolonged follow-up in the clinic, and should not manipulate the lenses himself. He should be told that any discomfort or change in vision needs urgent attention.

In summary, therefore, hard lenses remain the contact lenses of choice for the ordinary phakic wearer with no disease. Soft lenses should be reserved for those who cannot tolerate hard lenses and for those who want to wear lenses intermittently. Extended-wear, sadly the most attractive feature of soft lens philosophy, is wise only if the patient follows instructions closely and has immediate access to skilled advice when something seems to be wrong. For aphakic patients spectacles are much safer. Nevertheless, the use of soft lenses should be explored further, if only to emphasise that we need to evaluate intraocular acrylic implants much more critically.

\section{Foreign bodies in the rectum}

The eight external orifices of the human body seem to attract strange foreign bodies just as honey does bees. For size and variety of objects, pride of place goes to the rectum; but the circumstances responsible are varied. Firstly, thermometers, enema tips, and catheters may disappear within the rectum, and inspissated masses of barium may be left behind after radiological examination. Next is the therapeutic group: to relieve pruritus and prolapsing piles patients may use all sorts of blunt objects up to lamp bulbs, bottles, and broomstick handles, and indeed several old-fashioned proprietary "treatments" for haemorrhoids consisted of obturators for insertion into the anal canal. Criminal assault is an occasional cause, and another source of trouble is swallowing sharp foreign bodies which then impact in the lower rectum to present as either an abscess or a fistula. Finally - and today it seems most common-there is the introduction of a wide variety of objects into the rectum for sexual gratification. Haft and colleagues ${ }^{1}$ have recently reported two examples of women who presented with battery-driven vaginal vibrators lodged in the rectum during intercourse. One was removed per anum but the other required a laparotomy to dislodge the vibrator from the sigmoid colon into the rectal ampulla; in this instance the patient reported that the motor had continued to operate for five hours after insertion, surely a tribute to modern electric batteries.
Most reports of rectal foreign bodies are anecdotal and many more are simply recounted at medical mess dinners. A useful service has therefore been performed by Eftaiha and his colleagues ${ }^{2}$ from the section of colon and rectal surgery at Cook County Hospital, Chicago (one of the largest hospitals in the world), who recently reviewed a five-year experience of the removal of 31 colorectal foreign bodies in 30 patients, all men. They suggest a classification according to physical properties (sharp objects, large round foreign bodies, and glass) and location (whether easily palpable in the rectal ampulla or out of reach in or proximal to the rectosigmoid junction). After clinical examination, radiographs were taken of the abdomen and pelvis in two planes in order to establish the type, number, and location of the objects. Low-lying foreign bodies were removed transanally under spinal or local infiltration anaesthesia, which allowed complete relaxation of the anal sphincter. Sharp objects (bone spicules, toothpicks, glass fragments, etc) were delivered through a proctoscope with every care to prevent further mucosal laceration. Large rounded objects were easily removed with the help of forceps when there was no risk of breaking the foreign body, but glass containers required special care to avoid fracture. The suction effect created by the upward direction of the mouth of the container was ingeniously released by the use of a couple of Foley catheters passed around the container and extended into the lumen of the bowel above the foreign object. After inflating the balloons of the catheters, air was injected. Applying traction to the catheters will also help in removal of the object. Breaking the suction effect of the glass container in this manner will usually be successful. Others have also described the use of obstetric forceps to remove bottles in the rectum. ${ }^{34}$

For high-lying foreign bodies the Chicago group advocate spinal anaesthesia. The patient is placed in the lithotomy position, the object is located through the sigmoidoscope and manipulated, if possible, by abdominal palpation into the rectal ampulla. In three patients, however, laparotomy proved necessary. In one the object could then be manipulated into the rectum and removed, but in two others colotomy was needed, one to remove a ballpoint pen and the other to evacuate a large triangular-shaped glass bottle. The introduction of more and more ingenious attachments to the flexible fibreoptic colonoscope will no doubt reduce still further the need to resort to laparotomy for removal of highly situated objects.

After removal of a foreign body sigmoidoscopy should be carried out to exclude mucosal lacerations, perforation, or a missed second foreign body, and the patient should remain in hospital for a day or two postoperatively to be observed for delayed symptoms and signs of perforation or of perirectal suppuration.

${ }^{1}$ Haft, J S, Benjamin, H B, and Wagner, M, British Medical fournal, $1976,1,626$.

2 Eftaiha, M, Hambrick, E, and Abcarian, H, Archives of Surgery, 1977, 112, 691.

${ }^{3}$ Moynihan, N H, and Thomas, S H, St Thomas's Hospital Gazette, 1951, 49, 152.

${ }^{4}$ Peet, T N D, British Medical fournal, 1976, 1, 500. 